\title{
Improving Outcomes in Colorectal Surgery by Sequential Implementation of Multiple Standardized Care Programs
}

\author{
Jeffrey E Keenan, MD, Paul J Speicher, MD, MHSc, Daniel P Nussbaum, MD, \\ Mohamed Abdelgadir Adam, MD, Timothy E Miller, MB ChB, FRCA, \\ Christopher R Mantyh, MD, FACS, FASCRS, Julie KM Thacker, MD, FACS, FASCRS
}

BACKGROUND: The purpose of this study was to examine the impact of the sequential implementation of the enhanced recovery program (ERP) and surgical site infection bundle (SSIB) on short-term outcomes in colorectal surgery (CRS) to determine if the presence of multiple standardized care programs provides additive benefit.

STUDY DESIGN: Institutional ACS-NSQIP data were used to identify patients who underwent elective CRS from September 2006 to March 2013. The cohort was stratified into 3 groups relative to implementation of the ERP (February 1, 2010) and SSIB (July 1, 2011). Unadjusted characteristics and 30-day outcomes were assessed, and inverse proportional weighting was then used to determine the adjusted effect of these programs.

RESULTS: There were 787 patients included: 337, 165, and 285 in the pre-ERP/SSIB, post-ERP/preSSIB, and post-ERP/SSIB periods, respectively. After inverse probability weighting (IPW) adjustment, groups were balanced with respect to patient and procedural characteristics considered. Compared with the pre-ERP/SSIB group, the post-ERP/pre-SSIB group had significantly reduced length of hospitalization ( 8.3 vs 6.6 days, $\mathrm{p}=0.01)$ but did not differ with respect to postoperative wound complications and sepsis. Subsequent introduction of the SSIB then resulted in a significant decrease in superficial SSI $(16.1 \%$ vs $6.3 \%, \mathrm{p}<0.01)$ and postoperative sepsis $(11.2 \%$ vs $1.8 \%, \mathrm{p}<0.01)$. Finally, inflation-adjusted mean hospital cost for a CRS admission fell from $\$ 31,926$ in 2008 to $\$ 22,044$ in 2013 ( $\mathrm{p}<0.01$ ).

CONCLUSIONS: Sequential implementation of the ERP and SSIB provided incremental improvements in CRS outcomes while controlling hospital costs, supporting their combined use as an effective strategy toward improving the quality of patient care. (J Am Coll Surg 2015;221:404-414. (C) 2015 by the American College of Surgeons)

Surgeons are increasingly driving quality improvement efforts with the primary objective of improving patient safety and surgical outcomes. ${ }^{1,2}$ Furthermore, public

Disclosure Information: Nothing to disclose.

Disclosures outside the scope of this work: Dr Miller is a paid consultant to Edwards Lifesciences and has received grants from Covidien and Edwards Lifesciences. All other authors have nothing to disclose.

Presented at the American College of Surgeons National Surgical Quality Improvement Program National Conference, New York, NY, July 2014.

Received February 11, 2015; Revised April 8, 2015; Accepted April 8, 2015.

From the Departments of Surgery (Keenan, Speicher, Nussbaum, Adam, Mantyh, Thacker) and Anesthesiology (Miller), Duke University Medical Center, Durham, NC.

Correspondence address: Julie KM Thacker, MD, FACS, FASCRS, Department of Surgery, Duke University Medical Center, Box 2829, Durham, NC 27710. email: julie.thacker@dm.duke.edu reporting of hospital and surgeon outcomes data and pay-for-performance reimbursement schemes have added to the impetus to improve health care quality. ${ }^{3-5}$ In colorectal surgery (CRS), engaged surgeons have focused on implementing systems of care designed to reliably provide evidence-supported practices as a mechanism to improve postoperative outcomes. ${ }^{6}$ Two prominent systems of care are the enhanced recovery pathway $(\mathrm{ERP})^{7,8}$ and the preventive surgical site infection bundle (SSIB). The ERP is a comprehensive perioperative CRS care pathway that has been shown to reduce postoperative morbidity and length of hospitalization. ${ }^{9-17}$ Similarly, the SSIB is a perioperative care program that systematically provides evidence-supported measures for surgical wound infection prevention and has been shown to reduce the rate of postoperative wound infection. ${ }^{18-22}$ 


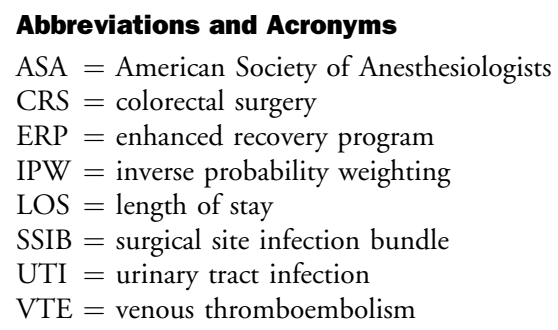

Although both the ERP and SSIB have individually been shown to improve outcomes after CRS, the effect of their combined presence has not, to our knowledge, previously been reported in the literature. The colorectal surgery service at our institution sequentially implemented the ERP in February 2010, followed by the SSIB in July 2011; together they have profoundly altered the practice of CRS at our institution. Previously, we had specifically evaluated the impact of SSIB on postoperative infection at our institution and found a significant reduction in the rate of SSI. ${ }^{22}$ In this study, we sought to build on this previous work by comprehensively evaluating the combined effect of the ERP and SSIB on the relevant short-term outcomes included in the American College of Surgeons (ACS) NSQIP in order to determine if the presence of these standardized care programs improved the quality and value of CRS care at our institution.

\section{METHODS}

\section{Patient selection and data collection}

This was a retrospective cohort study approved by the Institutional Review Board at Duke University Medical Center. Institutional ACS-NSQIP data files were used to identify a sample group of patients who underwent major CRS at Duke University Medical Center from September 1, 2006 through March 31, 2013 (Fig. 1). Participation in ACS-NSQIP provides institutions with data on a systematically sampled set of operations to serve as a vehicle for quality improvement. ${ }^{1,23,24}$ Specifically, every eighth operative day, demographic and clinical

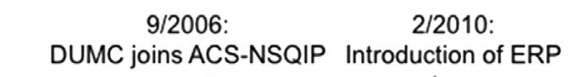

variables and 30-day outcomes on surgical patients are abstracted from the medical record by a trained surgical-clinical reviewer. The ACS-NSQIP system of data sampling has been validated, and data abstraction is routinely audited by outside surgical-clinical reviewers to ensure accuracy and consistency in data collection.

Procedures included in this study were low anterior resection, abdominoperineal resection, partial or total abdominal colectomy with or without proctectomy, proctectomy, pelvic exenteration, or Hartmann type procedure (CPT codes as follows: 44147, 44150-44151, 44160, 44204-44208, 44210, 44155-44158, 44211-44212, 45110-45114, 45116, 45119, 45120-45121, 45123, 45126, 45130, 45135, 45160, 45395, 45397, 45402, 45550). Both open and laparoscopic cases were included. Nonelective cases were excluded because the ERP was not commonly used in the urgent or emergent setting. Because the ERP and SSIB were used by the colorectal surgery group during the study period but not by other surgeons, only operations performed by board certified colorectal surgeons were included. During the study period, all procedures were performed by a total of 4 board certified colorectal surgeons who were part of a shared practice in 1 inpatient care facility, and all had 5 or more years of experience as attending surgeons at the starting point of their participation in the study.

Clinical data, including patient demographics, preoperative characteristics, operative factors, and 30-day outcomes, were determined using ACS-NSQIP institutional data files. Data for 30-day readmission as well as chemotherapy within 30 days of surgery were obtained by additional chart review because many of these data were missing due to changes in ACS-NSQIP data abstraction for these variables over the course of the study period. Data on compliance to specific components of the ERP were obtained from a prospectively maintained institutional database of patients treated under the ERP. Cost data, obtained from Duke University Hospital Finance, became available in 2008.

\section{Quality improvement programs}

During the study period, the ERP was introduced into practice by the colorectal surgery service on February 1,

7/2011:

Introduction SSIB

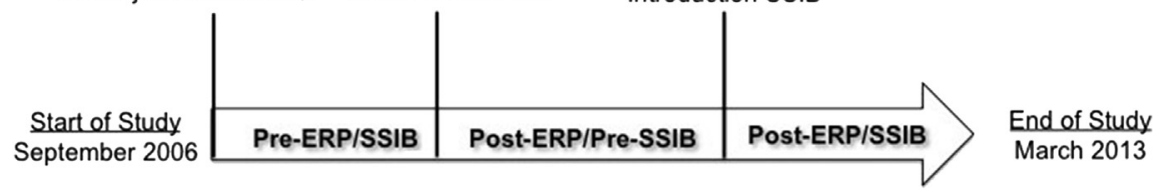

Figure 1. Schematic of the study period with key dates noted. DUMC, Duke University Medical Center; ACS NSQIP, American College of Surgeons National Surgery Quality Improvement Program; ERP, enhanced recovery pathway; SSIB, preventive surgical site infection bundle. 
2010. Our ERP is based largely on guidelines of the Enhanced Recovery After Surgery (ERAS) Society. ${ }^{7,8,13} \mathrm{Ma}-$ jor components of the ERP are shown in Figure 2. Briefly, in the preoperative period, measures are taken to ensure preoperative medical and nutritional optimization. To avoid acute dehydration and electrolyte disturbance, there is no overnight fast restriction, clear liquids are allowed up to 2 hours before surgery, and a carbohydrate-rich beverage is given in the preoperative holding area. ${ }^{25,26} \mathrm{An}$ xiolytics are avoided and a multimodal approach to postoperative nausea and vomiting is used. ${ }^{27}$ A prophylactic dose of unfractionated or low molecular weight heparin is given to reduce risk of venous thromboembolism (VTE) ${ }^{28}$ Intraoperatively, goal-directed fluid administration is used with esophageal Doppler or noninvasive hemodynamic monitoring devices, ${ }^{29-31}$ and minimally invasive surgery is used whenever possible. Analgesia is administered through a multimodal approach, including an epidural catheter or regional block for all patients. ${ }^{32}$ Postoperatively, the patient is immediately allowed a diet, and efforts are made to mobilize the patient on postoperative day zero. ${ }^{33}$ The Foley catheter is routinely removed by postoperative day 2 or earlier, even if an epidural catheter remains in place. The only exception to this protocol is when the attending physician specifically requests that the Foley catheter remain in place beyond postoperative day 2 , typically for cases that involve a low pelvic dissection. There is no routine administration of maintenance intravenous fluids, and efforts are made to minimize intravenous systemic narcotics. ${ }^{34}$

The patient is deemed suitable for discharge home when oral intake is adequate to maintain hydration, pain control is adequate with oral analgesics, and the patient can ambulate safely. Before discharge, the patient is counseled to provide appropriate postdischarge expectations and an understanding of issues that would require contacting the surgical team or presentation to the emergency department. Finally, in addition to receiving postoperative VTE prophylaxis while in the hospital, the patient is prescribed VTE prophylaxis for 4 weeks at home after discharge. ${ }^{35}$

The ERP represents a multidisciplinary approach, calling on surgeons, anesthesiologists, clinic nurses, operating room staff, mid-level providers, house staff, and nurses on the wards to enact its elements across all phases of perioperative care. Implementation and progress of the program were overseen by one of the colorectal surgeons, who met monthly with key personnel from each discipline to receive feedback on delivery of ERP elements, encourage compliance with ERP measures, and review recent outcomes data. Additionally, a prospectively maintained institutional database of patients treated under the ERP has been maintained in order to closely monitor adherence to prescribed ERP elements and to monitor outcomes.

\begin{tabular}{|c|c|c|c|}
\hline & PREOPERATIVE & INTRAOPERATIVE & POSTOPERATIVE \\
\hline 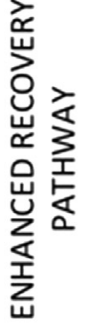 & $\begin{array}{l}\text { - } \quad \text { Education \& counseling } \\
\text { - } \quad \text { Medical optimization } \\
\text { - Nutritional optimization } \\
\text { - } \quad \text { Carbohydrate beverage two } \\
\text { hours prior to surgery } \\
\text { - } \quad \text { Multimodal prevention of PONV } \\
\text { - Avoidance of anxiolytics } \\
\text { - } \\
\text { Avoidance of sedatives } \\
\end{array}$ & $\begin{array}{l}\text { - Goal-directed fluid administration } \\
\text { - Minimally invasive surgery } \\
\text { epidtural analgesia or regional } \\
\text { blocks } \\
\text { - No routine peritoneal drains } \\
\text { - Removal of gastric tube in OR } \\
\text { Removal of urinary catheter in OR } \\
\text { or per epidural protocol }\end{array}$ & 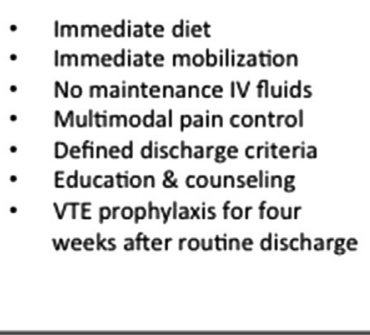 \\
\hline 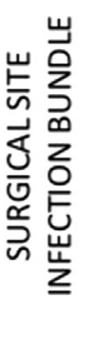 & $\begin{array}{l}\text { Mechanical bowel prep with oral } \\
\text { antibiotics night prior to surgery } \\
\text { - Chlorhexidine wipes in the } \\
\text { preoperative holding area } \\
\text { - Ertapenum within one hour of } \\
\text { incision } \\
\text { - Standardized surgical field } \\
\text { preparation with chlorhexidine- } \\
\text { isopropanol } \\
\text { - Standardized urinary catheter } \\
\text { placement }\end{array}$ & $\begin{array}{l}\text { - Limited OR traffic } \\
\text { Maintenance of euglycemia and } \\
\text { normothermia } \\
\text { - Fascial wound protector } \\
\text { Gown \& glove change prior to } \\
\text { fascial and skin closure } \\
\text { Dedicated wound closure tray }\end{array}$ & $\begin{array}{l}\text { Maintenance of euglycemia } \\
\text { and normothermia } \\
\text { - Removal of sterile dressing } \\
\text { within } 48 \text { hours } \\
\text { Daily washings of incision with } \\
\text { chlorhexidine for one week }\end{array}$ \\
\hline
\end{tabular}

Figure 2. Schematic of the components of the enhanced recovery pathway and preventive surgical site infection bundle across the phases of perioperative care. OR, operating room; PONV, postoperative nausea and vomiting; VTE, venous thromboembolism. 
The SSIB was introduced into practice by the CRS service on July 1, 2011. Key components of the SSIB are shown in Figure 2. Additionally, full details on the delivery and specific aspects of the SSIB have been described previously. ${ }^{22}$

\section{Analysis}

We first stratified patients into 3 groups defined by the timing of implementation of the ERP and SSIB as follows: pre-ERP/SSIB (September 2006 to January 2010); post-ERP/pre-SSIB (February 2010 to June 2011); and post-ERP/SSIB (July 2011 to March 2013). The primary outcomes measures were hospital length of stay (LOS) and wound complications. Secondary outcomes included 30-day mortality, 30-day readmission, unplanned reoperation rates, and the various organ-specific complications captured by ACS-NSQIP. Baseline characteristics and unadjusted outcomes were compared between groups using Pearson's chi-squared test or Fisher's exact test for categorical variables, and 1-way analysis of variance (ANOVA) for continuous variables.

In order to better estimate the effect of these individual programs on study outcomes, we performed adjusted comparisons of the pre-ERP/SSIB vs the post-ERP/preSSIB groups and the post-ERP/pre-SSIB vs the postERP/SSIB groups. Specifically, propensity scores were developed to control for potentially confounding variables. Covariates included in the propensity score model were age, sex, body mass index, American Society of Anesthesiologists (ASA) classification, type of colorectal procedure, use of laparoscopy, wound classification, and use of preoperative radiation. Using inverse probability weighting (IPW), the treatment effects of the ERP and SSIB were then estimated with weighted comparisons between the pre-ERP/SSIB vs the post-ERP/pre-SSIB groups and the post-ERP/pre-SSIB vs the post-ERP/ SSIB groups, respectively.

We also assessed adherence to the ERP by determining the compliance rates of specific program elements available in our institutional ERP database. Available elements included pre- and postoperative administration of VTE prophylaxis, use of intraoperative esophageal Doppler on noninvasive hemodynamic monitoring for goaldirected fluid administration, use of epidural analgesia and multimodal pain control, and ambulation on postoperative day 1.

Finally, available cost data were assessed to determine if there was any change in the cost of care associated with the introduction of the ERP and SSIB. The nominal total hospital cost data for each patient were converted into 2013 US dollars using the US Department of Labor consumer price index for hospital and related services. ${ }^{36}$
These costs were then stratified by year and assessed by the Cochran-Armitage trend test.

We made an affirmative decision to control for type I error at the level of the comparison, and a p value $<$ 0.05 was considered statistically significant. Propensity scores and IPW analyses were conducted using the R Toolkit for Weighting and Analysis of Nonequivalent Groups (TWANG), and all statistical analyses were performed using R version 3.0.2 (R Foundation for Statistical Computing).

\section{RESULTS}

There were 787 patients included in the study, with 337, 165 , and 285 in the pre-ERP/SSIB, post-ERP/pre-SSIB, and post-ERP/SSIB time periods, respectively (Table 1 ). These groups were balanced with respect to many characteristics including age, sex, race, BMI, wound classification, diabetes, COPD, smoking status, functional status, and preoperative radiation and chemotherapy. However, significant differences existed between the time periods in regard to type of procedure, ASA classification, and use of laparoscopy. The proportion of patients classified as ASA class 3 or greater appeared lower in the preERP/SSIB period than the later periods $(49.3 \%$ vs $68.5 \%$ vs $59.2 \%, \mathrm{p}<0.001$ ), and the proportion of laparoscopic cases increased considerably during the study period $(36.8 \%$ vs $46.1 \%$ vs $60 \%$, p $<0.001)$.

Evaluation of unadjusted 30-day postoperative outcomes revealed, respectively, a significant improvement in the mean ( 8.1 vs 6.6 vs 5.8 days, $\mathrm{p}<0.01)$ and median (6 vs 5 vs 5 days, $\mathrm{p}<0.01$ ) LOS over the study period, without a significant change in the rate of 30 -day readmission. There were also improvements observed for the rates of overall wound complication $(28.5 \%$ vs $19.4 \%$ vs $7.7 \%$, $\mathrm{p}<0.01)$, superficial SSI $(24.6 \%$ vs $18.8 \%$ vs $6.3 \%$, p < $0.01)$, organ-space SSI $(8.3 \%$ vs $6.1 \%$ vs $2.8 \%$, p $=$ $0.01)$, sepsis ( $8.9 \%$ vs $12.1 \%$ vs $1.8 \%, \mathrm{p}<0.01)$, and urinary tract infection (UTI) $(7.4 \%$ vs $5.5 \%$ vs $2.8 \%, \mathrm{p}=$ 0.04 ) over the course of the study period (Table 2). With the exception of postoperative sepsis, presence of the ERP alone appeared to improve these outcomes. Subsequent addition of the SSIB seemed then to increase the magnitude of improvement for many of the outcomes assessed.

Recognizing that there were important differences in the characteristics of the 3 time periods, which may have confounded the results, comparisons were performed using IPW in order to estimate the adjusted effects of the ERP and SSIB on postoperative outcomes. Following IPW, baseline characteristics of the pre-ERP/SSIB vs the post-ERP/pre-SSIB groups and the post-ERP/pre-SSIB 
Table 1. Cohort Characteristics Stratified by the Presence of the Enhanced Recovery Pathway and Preventive Surgical Site Infection Bundle

\begin{tabular}{|c|c|c|c|c|}
\hline Characteristic & $\begin{array}{c}\text { Pre-ERP/SSIB Sept } \\
2006-\text { Jan } 2010 \\
(n=337) \\
\end{array}$ & $\begin{array}{c}\text { Post-ERP/Pre-SSIB Feb } \\
\text { 2010-June 2011 } \\
\text { (n = 165) } \\
\end{array}$ & $\begin{array}{c}\text { Post-ERP/SSIB July } \\
\text { 2011-March 2013 } \\
(n=285) \\
\end{array}$ & p Value \\
\hline Age, y, mean $\pm S D$ & $59.9 \pm 14.6$ & $58.4 \pm 14.4$ & $57.0 \pm 17.0$ & 0.08 \\
\hline Sex, male, n (\%) & $159(47.2)$ & $73(44.2)$ & $141(49.5)$ & 0.56 \\
\hline Race, n (\%) & & & & 0.49 \\
\hline White & $266(78.9)$ & $122(73.9)$ & $217(76.1)$ & \\
\hline Black & $61(18.1)$ & $40(24.2)$ & $62(21.8)$ & \\
\hline Other & $10(3)$ & $3(1.8)$ & $6(2.1)$ & \\
\hline BMI, kg/m², median (Q1, Q3) & $28(24,32)$ & $28(24,32)$ & $27(24,31)$ & 0.07 \\
\hline ASA class $\geq 3, \mathrm{n}(\%)$ & $166(49.3)$ & $113(68.5)$ & $168(59.2)$ & $<0.01$ \\
\hline Procedure type, n (\%) & & & & $<0.01$ \\
\hline Partial colectomy & $107(31.9)$ & $62(37.6)$ & $115(40.5)$ & \\
\hline Hartmann & $6(1.8)$ & $0(0)$ & $6(2.1)$ & \\
\hline TAC without proctectomy & $17(5.1)$ & $15(9.1)$ & $16(5.6)$ & \\
\hline TAC with proctectomy & $16(4.8)$ & $11(6.7)$ & $26(9.2)$ & \\
\hline LAR & $119(35.5)$ & $61(37)$ & $94(33.1)$ & \\
\hline APR & $42(12.5)$ & $10(6.1)$ & $14(4.9)$ & \\
\hline Proctectomy (partial) & $26(7.8)$ & $5(3)$ & $11(3.9)$ & \\
\hline Pelvic exenteration & $2(0.6)$ & $1(0.6)$ & $2(0.7)$ & \\
\hline Laparoscopic procedure, $\mathrm{n}(\%)$ & $124(36.8)$ & $76(46.1)$ & $171(60)$ & $<0.01$ \\
\hline Wound classification, n (\%) & & & & 0.12 \\
\hline Clean/contaminated & $283(84)$ & $147(89.1)$ & $227(79.6)$ & \\
\hline Contaminated & $39(11.6)$ & $14(8.5)$ & $40(14)$ & \\
\hline Dirty/infected & $15(4.5)$ & $4(2.4)$ & $18(6.3)$ & \\
\hline Preoperative sepsis, n (\%) & $2(0.6)$ & $2(1.2)$ & $4(1.4)$ & 0.58 \\
\hline Diabetes, n (\%) & $47(13.9)$ & $29(17.6)$ & $33(11.6)$ & 0.21 \\
\hline COPD, n (\%) & $10(3)$ & $9(5.5)$ & $6(2.1)$ & 0.14 \\
\hline Smoker, n (\%) & $65(19.3)$ & $31(18.8)$ & $46(16.1)$ & 0.573 \\
\hline Independent functional status, n (\%) & $325(96.4)$ & $160(97)$ & $282(98.9)$ & 0.12 \\
\hline Chemotherapy $\leq 30 \mathrm{~d}, \mathrm{n}(\%)$ & $2(0.6)$ & $1(0.6)$ & $0(0.0)$ & 0.44 \\
\hline Radiotherapy $\leq 90 \mathrm{~d}, \mathrm{n}(\%)$ & $61(18.1)$ & $31(18.8)$ & $26(11.7)$ & 0.08 \\
\hline
\end{tabular}

vs the post-ERP/SSIB groups were balanced with respect to all characteristics considered (Table 3) (Supplemental Figures 1 and 2 show standardized differences between the unweighted and weighted groups). Compared with the pre-ERP/SSIB period, the post-ERP/pre-SSIB period demonstrated significant improvement in mean LOS (8.3 vs 6.6 days, $\mathrm{p}=0.01)$ as well as in the rate of unplanned reoperation $(7.6 \%$ vs $2.4 \%, p=0.03)$ (Table 4$)$. There was also a nonsignificant increase in the rate of 30-day readmission, and there were nonsignificant decreases in in the rate of wound complication and SSI in the postERP/pre-SSIB period. When comparing the IPWadjusted post-ERP/pre-SSIB and post-ERP/SSIB groups, there were significant reductions in the rate of overall wound complication $(16.7 \%$ vs $7.7 \%, \mathrm{p}=0.01)$, superficial SSI $(16.1 \%$ vs $6.3 \%, \mathrm{p}<0.01)$, and sepsis $(11.2 \%$ vs $1.8 \%, \mathrm{p}<0.01)$, respectively. There was also a significant decrease in the rate of 30-day readmission from the post-ERP/pre-SSIB to post-ERP/SSIB period $(18.2 \%$ vs $10.2 \%, \mathrm{p}=0.03)$, although the rate of readmission in the post-ERP/SSIB period appeared similar to that of the pre-ERP/SSIB period. Lastly, after adjustment with IPW, there were no longer significant differences in the rate of stroke and deep venous thrombosis observed between the different time periods.

To assess adherence to measures of the ERP, our institutional ERP database was queried. In the post-ERP/SSIB group, 223 of the 285 patients were contained within the ERP database. Of these patients, $90 \%$ received preoperative VTE prophylaxis, 98\% received postoperative VTE 
Table 2. 30-Day Postoperative Outcomes Stratified by the Presence of the Enhanced Recovery Pathway and Preventive Surgical Site Infection Bundle

\begin{tabular}{|c|c|c|c|c|}
\hline 30-day postoperative outcome & $\begin{array}{c}\text { Pre-ERP } / \text { SSIB, } \\
\text { Sept 2006-Jan } 2010 \\
(n=337)\end{array}$ & $\begin{array}{c}\text { Post-ERP/Pre-SSIB, } \\
\text { Feb 2010-June 2011 } \\
(n=165)\end{array}$ & $\begin{array}{c}\text { Post-ERP/SSIB, } \\
\text { July 2011-March } 2013 \\
(\mathrm{n}=\mathbf{2 8 5}) \\
\end{array}$ & p Value \\
\hline Mortality, n (\%) & $2(0.6)$ & $1(0.6)$ & $4(1.4)$ & 0.61 \\
\hline Median length of stay, d, (Q1, Q3) & $6(4,8)$ & $5(4,8)$ & $5(3,7)$ & $<0.01$ \\
\hline Length of stay, d, (mean \pm SD) & $8.1 \pm 7.6$ & $6.6 \pm 4.5$ & $5.8 \pm 6.0$ & $<0.01$ \\
\hline Readmission, $\mathrm{n}(\%)$ & $41(12.2)$ & $29(17.6)$ & $29(10.2)$ & 0.07 \\
\hline Unplanned reoperation, n (\%) & $24(7.1)$ & $4(2.4)$ & $9(3.2)$ & 0.03 \\
\hline Wound complication, n (\%) & $96(28.5)$ & $32(19.4)$ & $22(7.7)$ & $<0.01$ \\
\hline Superficial SSI & $83(24.6)$ & $31(18.8)$ & $18(6.3)$ & $<0.01$ \\
\hline Deep SSI & $5(1.5)$ & $1(0.6)$ & $0(0)$ & 0.10 \\
\hline Organ space SSI & $28(8.3)$ & $10(6.1)$ & $8(2.8)$ & 0.01 \\
\hline Sepsis, n (\%) & $30(8.9)$ & $20(12.1)$ & $5(1.8)$ & $<0.01$ \\
\hline Septic shock, n (\%) & $8(2.4)$ & $2(1.2)$ & $1(0.4)$ & 0.09 \\
\hline Deep venous thrombosis, n (\%) & $3(0.9)$ & $7(4.2)$ & $0(0)$ & $<0.01$ \\
\hline Pulmonary embolism, n (\%) & $1(0.3)$ & $2(1.2)$ & $2(0.7)$ & 0.37 \\
\hline Stroke/CVA, n (\%) & $0(0)$ & $3(1.8)$ & $3(1.1)$ & 0.04 \\
\hline Unplanned reintubation, n (\%) & $9(2.7)$ & $4(2.4)$ & $4(1.4)$ & 0.54 \\
\hline Pneumonia, n (\%) & $10(3)$ & $5(3)$ & $3(1.1)$ & 0.20 \\
\hline Myocardial infarction, n (\%) & $2(0.6)$ & $3(1.8)$ & $4(1.4)$ & 0.38 \\
\hline Cardiac arrest, n (\%) & $0(0)$ & $0(0)$ & $1(0.4)$ & 0.57 \\
\hline Urinarytract infection, $\mathrm{n}(\%)$ & $25(7.4)$ & $9(5.5)$ & $8(2.8)$ & 0.04 \\
\hline
\end{tabular}

CVA, cerebrovascular accident; ERP, enhanced recovery pathway; Q1, quartile 1; Q3, quartile 3; SSI, surgical site infection; SSIB, surgical site infection bundle.

prophylaxis, 95\% had goal-directed intraoperative fluid administration guided by either esophageal Doppler or noninvasive hemodynamic monitoring, $93 \%$ had an epidural and multimodal pain management, and $61 \%$ had documented ambulation on postoperative day 1 (Fig. 3).

Finally, available hospital financial data were evaluated to assess whether the introduction of the ERP and SSIB were associated with a reduction in hospital costs for CRS patients. After inflation adjustment, mean total hospital costs per CRS admission demonstrated a significant downward trend over this time period, falling from $\$ 31,926$ in 2008 to $\$ 22,044$ in 2013 ( $\mathrm{p}<0.001$ ) (Fig. 4).

\section{DISCUSSION}

Over the past 5 years, the colorectal surgery service at our institution has substantially changed its practice by implementing the ERP and SSIB quality improvement initiatives. Building on our previous work, in which we specifically examined the impact of the SSIB on postoperative SSI, ${ }^{22}$ in this study, we sought to comprehensively evaluate how the sequential implementation of the ERP followed by the SSIB affected CRS outcomes at our institution in order to determine whether the presence of multiple standardized care programs can provide additive improvement in care quality and patient safety. Toward this end, we stratified the cohort into 3 groups based on the time of implementation of the ERP and SSIB in order to investigate whether outcomes improved in relation to the advent of these respective standardized care programs. In the adjusted analysis, we found that the introduction of the ERP resulted in reduced LOS and reduced rates of superficial and organ space SSI, and UTI. Subsequent addition of the SSIB appeared to augment the improvements observed with the ERP alone with respect to LOS, superficial and organ space SSI, and UTI, while also offering additional benefit through reduced rates of sepsis.

In an attempt to control for differences in patient and procedural characteristics between the different time periods, we also conducted adjusted comparisons of the pre-ERP/SSIB vs the post-ERP/pre-SSIB and the post$\mathrm{ERP} /$ pre-SSIB vs the post-ERP/SSIB time periods using IPW. This analysis demonstrated that introduction of the ERP led to a significant decrease in LOS and unplanned reoperation. In turn, subsequent addition of the SSIB led to significant reduction in the rate of postoperative wound complications, superficial SSI, and sepsis. 
Table 3. Baseline Characteristics of the Pre-ERP/SSIB vs Post-ERP/Pre-SSIB and Post-ERP/Pre-SSIB vs Post-ERP/SSIB Groups after Inverse Proportional Weighting

\begin{tabular}{|c|c|c|c|c|c|c|c|c|}
\hline \multirow[b]{2}{*}{ Characteristic } & \multicolumn{4}{|c|}{ Pre-ERP/SSIB vs Post-ERP/Pre-SSIB } & \multicolumn{4}{|c|}{ Post-ERP/Pre-SSIB vs Post-ERP/SSIB } \\
\hline & $\begin{array}{l}\text { Pre-ERP/ } \\
\text { SSIB }\end{array}$ & $\begin{array}{c}\text { Post-ERP/ } \\
\text { Pre-SSIB } \\
\end{array}$ & $\begin{array}{c}\text { Standard } \\
\text { difference, \%* }\end{array}$ & $\begin{array}{c}p \\
\text { Value }\end{array}$ & $\begin{array}{c}\text { Post-ERP/ } \\
\text { Pre-SSIB } \\
\end{array}$ & $\begin{array}{c}\text { Post-ERP/ } \\
\text { SSIB }\end{array}$ & $\begin{array}{c}\text { Standard } \\
\text { difference, \%* }\end{array}$ & p Value \\
\hline Age, y, mean $\pm S D$ & $58.3 \pm 14.3$ & $58.4 \pm 14.4$ & 0.9 & 0.93 & $57.4 \pm 15.7$ & $57 \pm 17$ & 2.7 & 0.80 \\
\hline Male sex, \% & 50.3 & 44.2 & 12.3 & 0.25 & 41.4 & 49.5 & 16.2 & 0.11 \\
\hline Race, \% & & & & 0.53 & & & & 0.89 \\
\hline White & 76.5 & 73.9 & 5.8 & & 74.2 & 76.1 & 4.6 & \\
\hline Black & 20.4 & 24.2 & 8.9 & & 23.8 & 21.8 & 5.0 & \\
\hline Other & 3.1 & 1.8 & 9.6 & & 2.0 & 2.1 & 0.5 & \\
\hline $\mathrm{BMI}, \mathrm{kg} / \mathrm{m}^{2}$, median $\pm \mathrm{SD}$ & $27.9 \pm 5.8$ & $27.9 \pm 5.8$ & 0.6 & 0.95 & $27.8 \pm 5.9$ & $27.7 \pm 5.9$ & 1.8 & 0.86 \\
\hline ASA class $\geq 3, \%$ & 65.7 & 68.5 & 5.9 & 0.56 & 61.5 & 59.2 & 4.8 & 0.65 \\
\hline Procedure type, $\%$ & & & & 0.85 & & & & 0.56 \\
\hline Partial colectomy & 36.9 & 37.6 & 1.3 & & 43.7 & 40.5 & 6.6 & \\
\hline Hartmann & 1.1 & 0.0 & 0.0 & & 0.0 & 2.1 & 14.7 & \\
\hline TAC without proctectomy & 7.5 & 9.1 & 5.6 & & 7.6 & 5.6 & 8.5 & \\
\hline TAC with proctectomy & 5.9 & 6.7 & 3.0 & & 6.9 & 9.2 & 7.8 & \\
\hline LAR & 38.9 & 37.0 & 4.0 & & 33.2 & 33.1 & 0.3 & \\
\hline APR & 6.3 & 6.1 & 1.1 & & 4.4 & 4.9 & 2.5 & \\
\hline Proctectomy (partial) & 3.0 & 3.0 & 0.3 & & 3.1 & 3.9 & 4.0 & \\
\hline Pelvic exenteration & 0.4 & 0.6 & 3.2 & & 1.0 & 0.7 & 3.9 & \\
\hline Laparoscopic procedure & 43.0 & 46.1 & 6.1 & 0.57 & 56.0 & 60.0 & 8.1 & 0.43 \\
\hline Wound classification, \% & & & & 0.83 & & & & 0.45 \\
\hline Clean/contaminated & 87.1 & 89.1 & 6.2 & & 83.7 & 79.6 & 10.2 & \\
\hline Contaminated & 9.9 & 8.5 & 5.2 & & 12.9 & 14.0 & 3.2 & \\
\hline Dirty/infected & 2.9 & 2.4 & 3.3 & & 3.3 & 6.3 & 12.3 & \\
\hline Preoperative sepsis, \% & 0.3 & 1.2 & 8.0 & 0.18 & 1.6 & 1.4 & 1.3 & 0.91 \\
\hline Diabetes, \% & 11.7 & 17.6 & 15.5 & 0.08 & 15.5 & 11.6 & 12.2 & 0.24 \\
\hline COPD, \% & 3.4 & 5.5 & 9.2 & 0.32 & 5.5 & 2.1 & 23.7 & 0.06 \\
\hline Smoker, \% & 21.8 & 18.8 & 7.8 & 0.47 & 18.2 & 16.1 & 5.7 & 0.58 \\
\hline Independent functional status, $\%$ & 95.9 & 97.0 & 6.0 & 0.62 & 97.3 & 98.9 & 16.5 & 0.18 \\
\hline Chemotherapy, $<30 \mathrm{~d}$, \% & 0.8 & 0.6 & 2.6 & 0.81 & 1.0 & 0.0 & 0.0 & 0.28 \\
\hline Radiotherapy, $<90$ d, \% & 18.0 & 18.8 & 2.1 & 0.83 & 12.8 & 10.9 & 6.3 & 0.51 \\
\hline
\end{tabular}

${ }^{*}$ Standard differences less than $20 \%$ indicate negligible differences between groups for a particular covariate.

APR, abdominoperineal resection; ASA, American Society of Anesthesiologists; ERP, enhanced recovery pathway; LAR, low anterior resection; SSIB, surgical site infection bundle; TAC, total abdominal colectomy.

Together, these findings suggest that the ERP and SSIB functioned in a complementary fashion, each individually imparting benefit with respect to different postoperative outcomes. Overall, it appears the sequential implementation of these programs has provided incremental improvement in patient care, even after accounting for differences in a number of patient and procedural characteristics between the different time periods.

In addition to investigating short-term outcomes after the advent of the ERP and SSIB, we evaluated compliance to specific enhanced recovery measures. The inability to achieve high compliance is commonly cited as a reason why quality improvement programs fail. ${ }^{6,18,37,38}$ To avoid this potential pitfall, elements of the ERP and SSIB have been protocolized and integrated into physician order sets, which has facilitated reliable delivery of these programs. Additionally, CRS leadership meets frequently with members of the multidisciplinary team that enacts the components of these programs in order to reinforce program objectives, identify problem areas, and improve program coordination. Reflecting these efforts, the compliance rate observed for pre- and postoperative VTE prophylaxis administration, intraoperative goaldirected fluid administration, and use of epidural analgesia with multimodal pain control was $90 \%$ or better. In addition, although the components of the SSIB program elements were not tracked within our ERP database, data available from the Surgery Care Improvement 
Table 4. Thirty-Day Postoperative Outcomes of the Pre-ERP/SSIB vs Post-ERP/Pre-SSIB and Post-ERP/Pre-SSIB vs PostERP/SSIB Groups after Inverse Proportional Weighting

\begin{tabular}{|c|c|c|c|c|c|c|}
\hline \multirow{2}{*}{$\begin{array}{l}\text { Inverse proportional weighting-adjusted } \\
\text { outcomes }\end{array}$} & \multicolumn{3}{|c|}{ Pre-ERP/SSIB vs Post-ERP/Pre-SSIB } & \multicolumn{3}{|c|}{ Post-ERP/Pre-SSIB vs Post-ERP/SSIB } \\
\hline & Pre-ERP/SSIB & Post-ERP/Pre-SSIB & p Value & Post-ERP/Pre-SSIB & Post-ERP/SSIB & p Value \\
\hline Mortality, \% & 0.7 & 0.6 & 0.94 & 0.7 & 1.4 & 0.53 \\
\hline Length of stay, $d$, mean $\pm S D$ & $8.3 \pm 8$ & $6.6 \pm 4.5$ & 0.01 & $6.5 \pm 4.9$ & $5.8 \pm 6$ & 0.18 \\
\hline Readmission, \% & 11.9 & 17.6 & 0.12 & 18.2 & 10.2 & 0.03 \\
\hline Unplanned reoperation, \% & 7.6 & 2.4 & 0.03 & 4.2 & 3.2 & 0.65 \\
\hline Wound complication, \% & 25.3 & 19.4 & 0.16 & 16.7 & 7.7 & 0.01 \\
\hline Superficial SSI & 21.4 & $18.8 \%$ & 0.52 & 16.1 & 6.3 & $\overline{0.001}$ \\
\hline Deep SSI & 2.0 & 0.6 & 0.25 & 0.7 & 0.0 & 0.29 \\
\hline Organ space SSI & 8.8 & 6.1 & 0.31 & 6.4 & 2.8 & 0.13 \\
\hline Sepsis, \% & 9.1 & 12.1 & 0.33 & 11.2 & 1.8 & $<0.001$ \\
\hline Septic shock, \% & 2.4 & 1.2 & 0.37 & 1.8 & 0.4 & 0.32 \\
\hline Deep venous thrombosis, $\%$ & 1.0 & 4.2 & 0.06 & 4.6 & 0.0 & 0.01 \\
\hline Pulmonary embolism, \% & 0.2 & 1.2 & 0.24 & 1.0 & 0.7 & 0.74 \\
\hline Stroke/CVA, \% & 0.0 & 1.8 & 0.08 & 2.4 & 1.1 & 0.42 \\
\hline Unplanned reintubation, \% & 2.7 & 2.4 & 0.87 & 3.4 & 1.4 & 0.29 \\
\hline Pneumonia, \% & 2.4 & 3.0 & 0.71 & 3.8 & 1.1 & 0.16 \\
\hline Myocardial infarction, $\%$ & 0.9 & 1.8 & 0.50 & 2.1 & 1.4 & 0.61 \\
\hline Cardiac arrest, $\%$ & 0.0 & 0.0 & 0.99 & 0.0 & 0.4 & 0.32 \\
\hline Urinary tract infection, $\%$ & 8.1 & 5.5 & 0.30 & 4.8 & 2.8 & 0.31 \\
\hline
\end{tabular}

CVA, cerebrovascular accident; ERP, enhanced recovery pathway; SSI, surgical site infection; SSIB, surgical site infection bundle.

Project ${ }^{39}$ have indicated that compliance with appropriate perioperative antibiotic administration as well as perioperative euglycemia and normothermia goals has been $95 \%$ or greater since introduction of the SSIB. It is worth noting that the initial establishment of a multidisciplinary structure for administration of the ERP greatly facilitated the subsequent introduction of the SSIB because the SSIB drew on essentially the same providers and support staff.

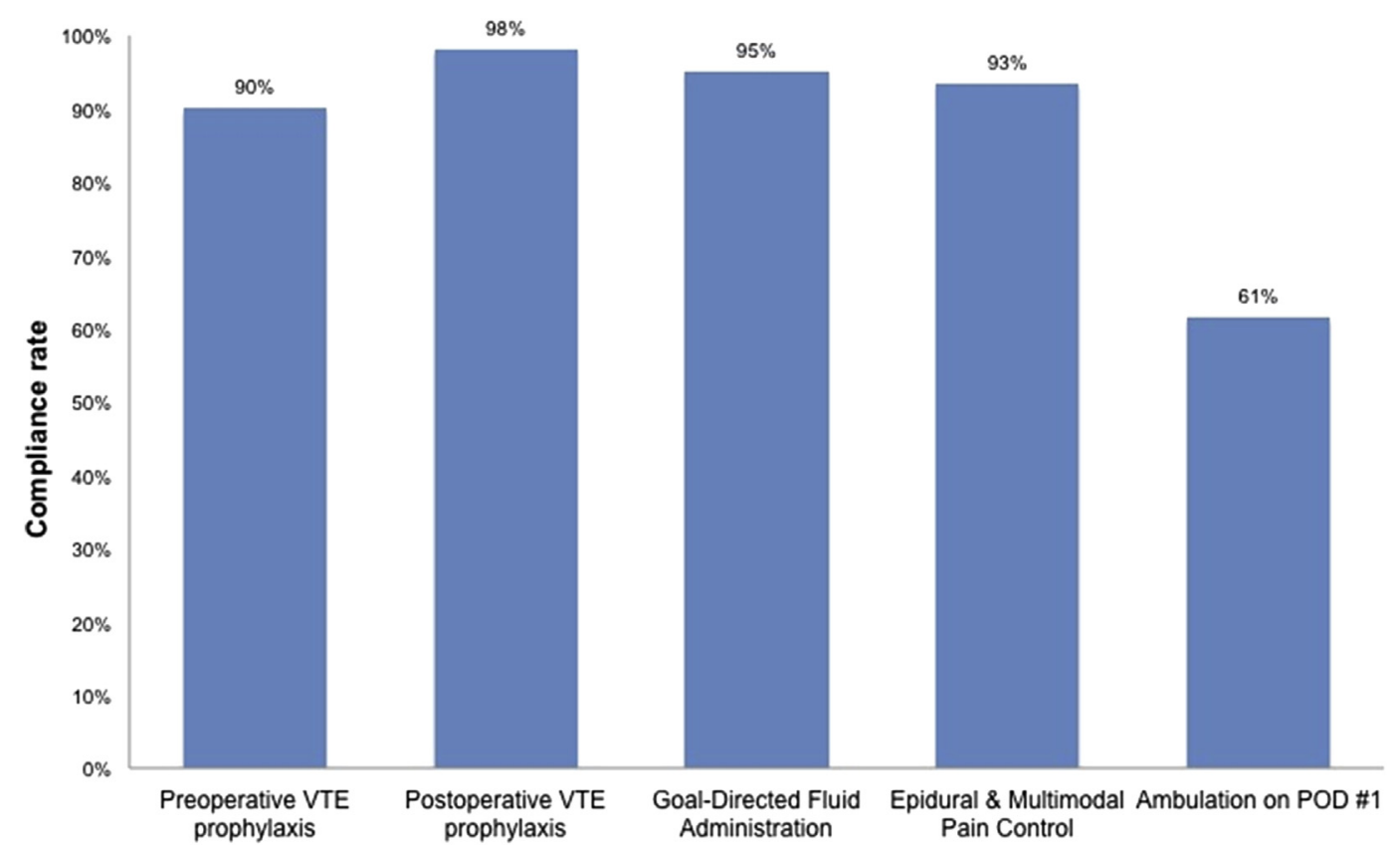

Figure 3. Compliance rate of specific enhanced recovery program measures captured in the institutional enhance recovery program database. VTE, venous thromboembolism; POD, postoperative day. 


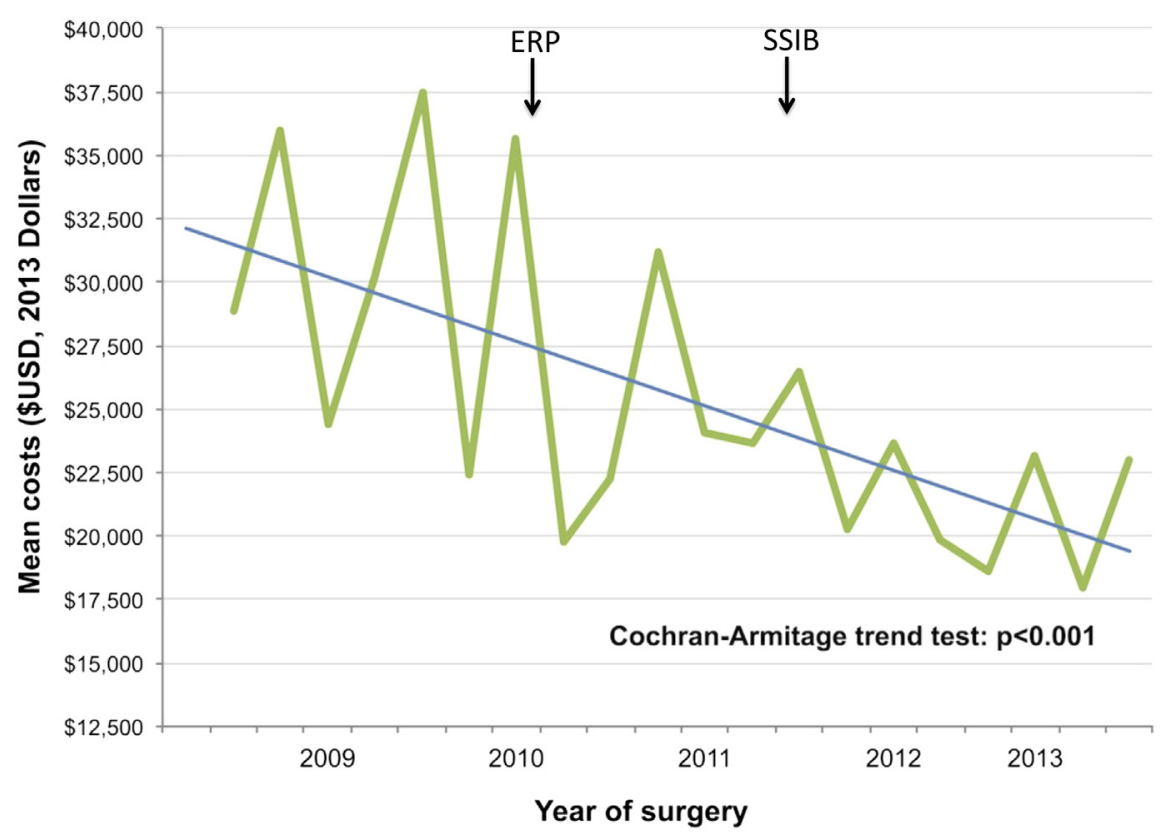

Figure 4. Mean total cost of hospitalization (green line) for colorectal surgery patients from 2008 to 2013, adjusted to 2013 US dollars. Time points of the introduction of the enhanced recovery pathway (ERP) and surgical site infection bundle (SSIB) are superimposed as indicated by the labeled black arrows. The trend of mean total cost with respect to time (blue line) was assessed by the Cochran-Armitage trend test.

Our experience has informed us that once a good structure for the systematic delivery of care across the perioperative phases is in place, the ability to implement additional programs is enhanced.

Finally, we examined costs of care during the period in which the ERP and SSI were introduced. Given the high and rising costs of health care, improving patient outcomes while also controlling costs is an important goal for quality improvement initiatives. ${ }^{40}$ To a large degree, the elements of the ERP and SSIB use existing hospital resources in a reliable, systematic manner without incurring new capital costs or using newer, more expensive technology. We therefore speculated that implementation of the ERP and SSIB would be associated with a reduction in hospital costs for a CRS admission. Indeed, the mean inflation-adjusted cost for a CRS admission decreased by nearly $\$ 10,000$ (2013 US dollars) from 2008 to 2013. So it appears that the ERP and SSIB were not only effective programs, but they also were associated with cost savings. When interpreting the changes in the cost of a CRS admission over the study period, it is important to consider that a myriad of complex factors affect the cost of health care delivery, including contracting rates with vendors, health system structure, and a variety of others. Such factors likely varied at least to some extent over the study period, and because this assessment of costs over time was not adjusted for these or patientlevel factors, we cannot necessarily assert that the ERP/ SSIB caused a reduction in costs. We only stipulate that costs savings per CRS admission were realized during the time period in which the ERP and SSIB were introduced, which may, at least in part, reflect increased value of care delivered due to improved outcomes and more efficient resource use brought about by these programs.

There are several limitations to our study that merit mention. First, we examined the effect of 2 quality improvement programs, each containing a number of interventions. Our reported results are those of the cumulative effect of these programs; we cannot determine which specific program elements were associated with benefits to a specific outcome. Second, as with any retrospective nonrandomized study, it is difficult to fully account for potentially confounding factors. The ACS-NSQIP data provide detailed and accurate information on a number of patients and operative variables, which were accounted for in our IPW-adjusted comparison. However, we acknowledge that there still may have been unmeasured variables that could not be adjusted for that may have influenced the study findings. Last, the study cohort was limited to elective CRS patients treated by a relatively small group of experienced colorectal surgeons at a single institution. The study findings may therefore not be generalizable 
to other institutions or surgical subspecialties. However, we anticipate, with appropriate field and institutionspecific modifications, that quality improvement programs like the ERP and SSIB can provide benefits in other surgical fields and institutions.

\section{CONCLUSIONS}

In conclusion, to our knowledge, this is the first study that demonstrates improved short-term outcomes of CRS with the combined presence of the ERP and SSIB. Our experience exemplifies how the process of quality improvement in surgery must be an ongoing effort, and that the sequential adoption of multiple standardized care protocols can provide incremental improvements in patient care while also helping to control health care costs. In the future, it will be important to continually assess and update care protocols such as the ERP and SSIB in order to include new evidence-based practices that can further improve patient safety and the value of care provided.

\section{Author Contributions}

Study conception and design: Keenan, Speicher, Nussbaum, Adam, Miller, Mantyh, Thacker

Acquisition of data: Keenan, Nussbaum, Adam

Analysis and interpretation of data: Keenan, Speicher, Nussbaum, Adam, Miller, Mantyh, Thacker

Drafting of manuscript: Keenan

Critical revision: Keenan, Speicher, Nussbaum, Adam, Miller, Mantyh, Thacker

\section{REFERENCES}

1. Ingraham AM, Richards KE, Hall BL, et al. Quality improvement in surgery: the American College of Surgeons National Surgical Quality Improvement Program approach. Adv Surg 2010;44:251-267.

2. Varkey P, Reller MK, Resar RK. Basics of quality improvement in health care. Mayo Clinic Proc 2007;82:735-739.

3. Jones RS, Brown C, Opelka F. Surgeon compensation: "Pay for performance," the American College of Surgeons National Surgical Quality Improvement Program, the Surgical Care Improvement Program, and other considerations. Surgery 2005;138:829-836.

4. Lindenauer PK, Remus D, Roman S, et al. Public reporting and pay for performance in hospital quality improvement. N Engl J Med 2007;356:486-496.

5. Ryan A, Blustein J. Making the best of hospital pay for performance. N Engl J Med 2012;366:1557-1559.

6. Arriaga AF, Lancaster RT, Berry WR, et al. The Better Colectomy Project: association of evidence-based best-practice adherence rates to outcomes in colorectal surgery. Ann Surg 2009;250:507-513.

7. Gustafsson UO, Scott MJ, Schwenk W, et al. Guidelines for perioperative care in elective colonic surgery: Enhanced Recovery After Surgery (ERAS(R)) Society recommendations. World J Surg 2013;37:259-284.
8. Nygren J, Thacker J, Carli F, et al. Guidelines for perioperative care in elective rectal/pelvic surgery: Enhanced Recovery After Surgery $(\operatorname{ERAS}(\mathrm{R}))$ Society recommendations. World J Surg 2013;37:285-305.

9. Basse L, Hjort Jakobsen D, Billesbolle P, et al. A clinical pathway to accelerate recovery after colonic resection. Ann Surg 2000;232:51-57.

10. Basse L, Raskov HH, Hjort Jakobsen D, et al. Accelerated postoperative recovery programme after colonic resection improves physical performance, pulmonary function and body composition. Br J Surg 2002;89:446-453.

11. Geltzeiler CB, Rotramel A, Wilson C, et al. Prospective study of colorectal enhanced recovery after surgery in a community hospital. JAMA Surg 2014;149:955-961.

12. Khoo CK, Vickery CJ, Forsyth N, et al. A prospective randomized controlled trial of multimodal perioperative management protocol in patients undergoing elective colorectal resection for cancer. Ann Surg 2007;245:867-872.

13. Miller TE, Thacker JK, White WD, et al. Reduced length of hospital stay in colorectal surgery after implementation of an enhanced recovery protocol. Anesth Analg 2014;118: 1052-1061.

14. Nicholson A, Lowe MC, Parker J, et al. Systematic review and meta-analysis of enhanced recovery programmes in surgical patients. Br J Surg 2014;101:172-188.

15. Serclova Z, Dytrych P, Marvan J, et al. Fast-track in open intestinal surgery: prospective randomized study (Clinical Trials Gov Identifier no. NCT00123456). Clin Nutr 2009;28:618-624.

16. Zhuang CL, Ye XZ, Zhang XD, et al. Enhanced recovery after surgery programs versus traditional care for colorectal surgery: a meta-analysis of randomized controlled trials. Dis Colon Rectum 2013;56:667-678.

17. Wind J, Hofland J, Preckel B, et al. Perioperative strategy in colonic surgery; Laparoscopy and/or Fast track multimodal management versus standard care (LAFA trial). BMC Surg 2006;6:16.

18. Bull A, Wilson J, Worth LJ, et al. A bundle of care to reduce colorectal surgical infections: an Australian experience. J Hosp Infect 2011;78:297-301.

19. Cima R, Dankbar E, Lovely J, et al. Colorectal Surgery Surgical Site Infection Reduction Program: A National Surgical Quality Improvement Program-driven multidisciplinary singleinstitution experience. J Am Coll Surg 2013;216:23-33.

20. Hedrick TL, Heckman JA, Smith RL, et al. Efficacy of protocol implementation on incidence of wound infection in colorectal operations. J Am Coll Surg 2007;205:432-438.

21. Wick EC, Hobson DB, Bennett JL, et al. Implementation of a surgical comprehensive unit-based safety program to reduce surgical site infections. J Am Coll Surg 2012;215:193-200.

22. Keenan JE, Speicher PJ, Thacker JK, et al. The Preventive Surgical Site Infection Bundle in Colorectal Surgery: An effective approach to surgical site infection reduction and health care cost savings. JAMA Surg 2014;149:1045-1052.

23. Khuri SF. Safety, quality, and the National Surgical Quality Improvement Program. Am Surg 2006;72:994-998; discussion 1021-1030, 1033-1048.

24. American College of Surgeons National Surgical Quality Improvement Program. Available at: http://site.acsnsqip.org/. Accessed March 14, 2015.

25. Noblett SE, Watson DS, Huong H, et al. Pre-operative oral carbohydrate loading in colorectal surgery: a randomized controlled trial. Colorectal Dis 2006;8:563-569. 
26. Practice guidelines for preoperative fasting and the use of pharmacologic agents to reduce the risk of pulmonary aspiration: application to healthy patients undergoing elective procedures: a report by the American Society of Anesthesiologist Task Force on Preoperative Fasting. Anesthesiology 1999;90:896-905.

27. Chandrakantan A, Glass PS. Multimodal therapies for postoperative nausea and vomiting, and pain. Brit J Anaesth 2011; 107[Suppl 1]:i27-i40.

28. Kwon S, Meissner M, Symons R, et al. Perioperative pharmacologic prophylaxis for venous thromboembolism in colorectal surgery. J Am Coll Surg 2011;213:596-603.e1.

29. Waldron NH, Miller TE, Thacker JK, et al. A prospective comparison of a noninvasive cardiac output monitor versus esophageal Doppler monitor for goal-directed fluid therapy in colorectal surgery patients. Anesth Analg 2014;118:966-975.

30. Pearse RM, Harrison DA, MacDonald N, et al. Effect of a perioperative, cardiac output-guided hemodynamic therapy algorithm on outcomes following major gastrointestinal surgery: a randomized clinical trial and systematic review. JAMA 2014; 311:2181-2190.

31. Noblett SE, Snowden CP, Shenton BK, Horgan AF. Randomized clinical trial assessing the effect of Doppler-optimized fluid management on outcome after elective colorectal resection. Br J Surg 2006;93:1069-1076.

32. Block BM, Liu SS, Rowlingson AJ, et al. Efficacy of postoperative epidural analgesia: a meta-analysis. JAMA 2003;290: $2455-2463$.
33. Karliczek A, Jesus EC, Matos D, et al. Drainage or nondrainage in elective colorectal anastomosis: a systematic review and meta-analysis. Colorectal Dis 2006;8:259-265.

34. Werawatganon T, Charuluxanun S. Patient controlled intravenous opioid analgesia versus continuous epidural analgesia for pain after intra-abdominal surgery. Cochrane Database Syst Rev 2005:CD004088.

35. Vedovati MC, Becattini C, Rondelli F, et al. A randomized study on 1-week versus 4-week prophylaxis for venous thromboembolism after laparoscopic surgery for colorectal cancer. Ann Surg 2014;259:665-669.

36. United States Department of Labor, Bureau of Labor Statistics. Consumer Price Index Databases. Available at http:// www.bls.gov/cpi/data.htm. Accessed July 16, 2014.

37. Pearsall EA, Meghji Z, Pitzul KB, et al. A qualitative study to understand the barriers and enablers in implementing an enhanced recovery after surgery program. Ann Surg 2014.

38. Cakir H, van Stijn MF, Lopes Cardozo AM, et al. Adherence to Enhanced Recovery After Surgery and length of stay after colonic resection. Colorectal Dis 2013;15:1019-1025.

39. Bratzler DW, Hunt DR. The surgical infection prevention and surgical care improvement projects: national initiatives to improve outcomes for patients having surgery. Clin Infect Dis 2006;43:322-330.

40. Lee L, Li C, Landry T, et al. A systematic review of economic evaluations of enhanced recovery pathways for colorectal surgery. Ann Surg 2014;259:670-676. 


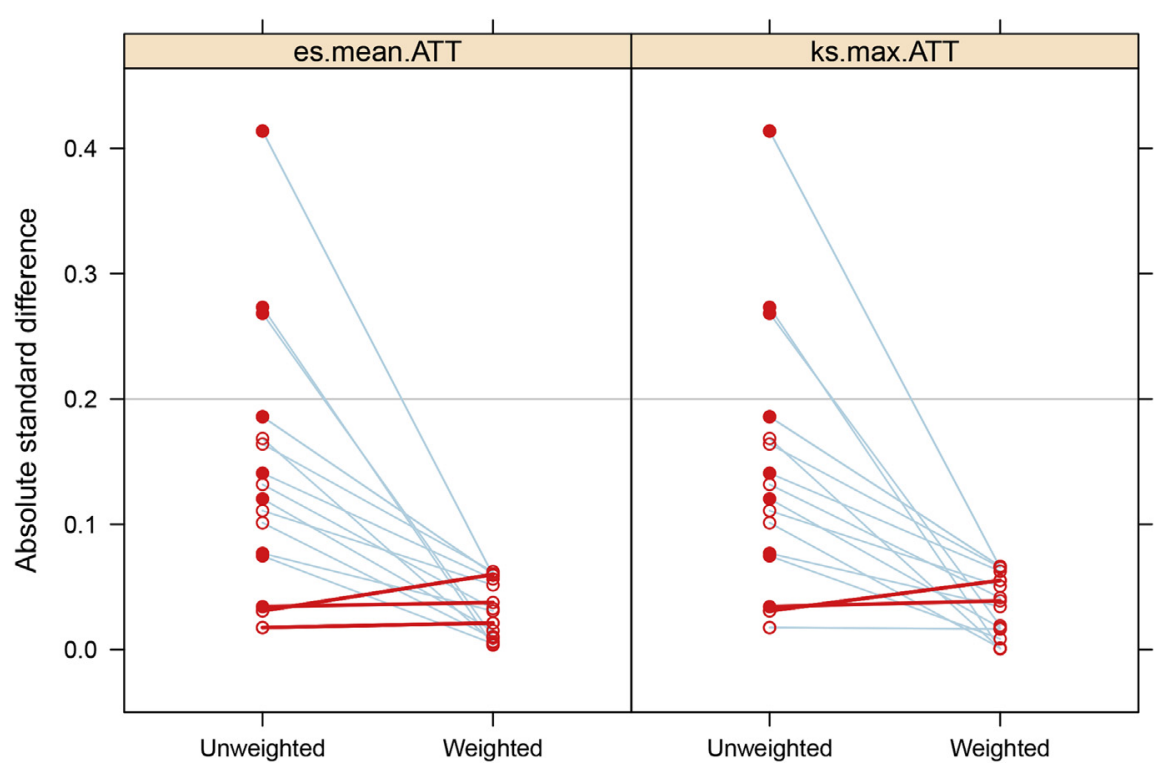

Supplemental Figure 1. Standardized differences in the pre-ERP/SSIB vs post-ERP/pre-SSIB baseline characteristics before (unweighted) and after (weighted) inverse probability weighting. Assessment of pre- and post-IPW standardized differences across variables using two stopping rules to assess balance on the covariates: es.mean.ATT, or the absolute standardized mean difference of the effect size, and ks.max.ATT, or the maximum Kolmogorov-Smirnov statistic across variables. ERP, enhanced recovery pathway; SSIB, surgical site infection bundle.

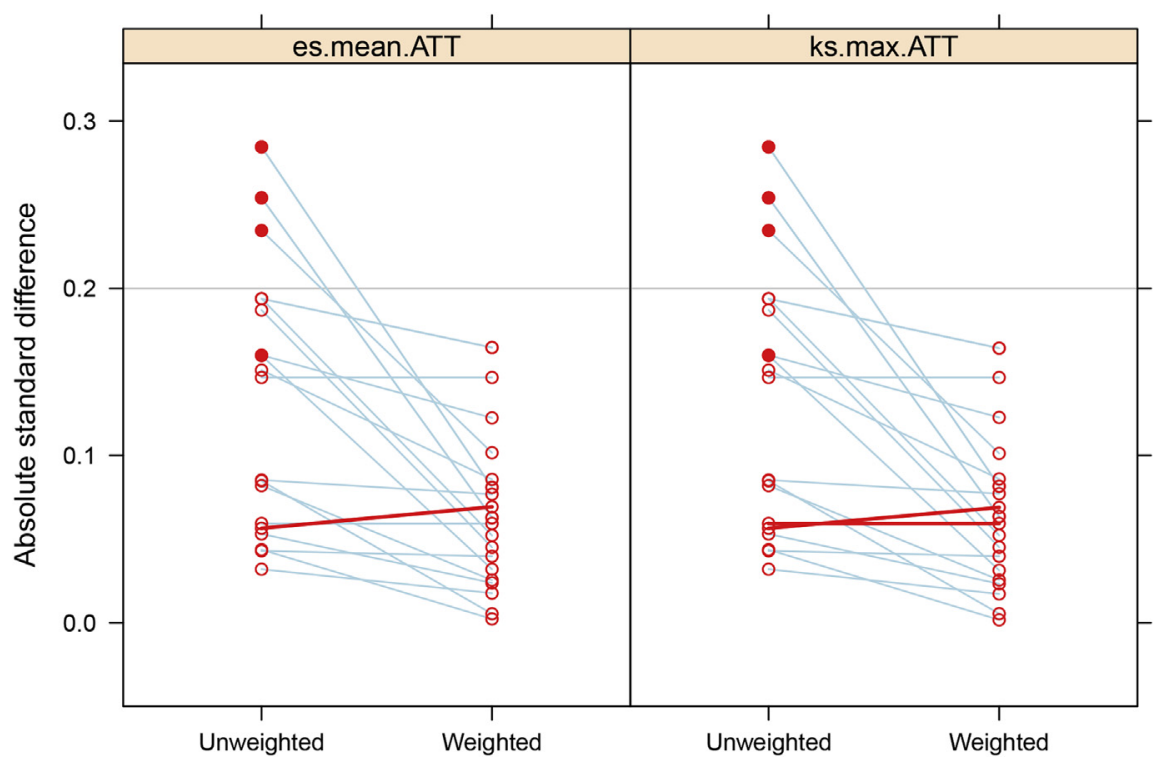

Supplemental Figure 2. Standardized differences in the post-ERP/pre-SSIB vs post-ERP/SSIB baseline characteristics before (unweighted) and after (weighted) inverse probability weighting. Assessment of pre- and post-IPW standardized differences across variables using two stopping rules to assess balance on the covariates: es.mean.ATT, or the absolute standardized mean difference of the effect size, and ks.max.ATT, or the maximum Kolmogorov-Smirnov statistic across variables. ERP, enhanced recovery pathway; SSIB, surgical site infection bundle. 